\title{
An Optimal Transmission Strategy for Joint Wireless Information and Energy Transfer in MIMO Relay Channels
}

\author{
Dingcheng Yang, Xiaoxiao Zhou, and Lin Xiao \\ Information Engineering School, Nanchang University, Nanchang 330031, China \\ Correspondence should be addressed to Dingcheng Yang; ydcxuanyuan@msn.com
}

Received 31 July 2014; Revised 29 December 2014; Accepted 8 January 2015

Academic Editor: Christoph F. Mecklenbräuker

Copyright ( 2015 Dingcheng Yang et al. This is an open access article distributed under the Creative Commons Attribution License, which permits unrestricted use, distribution, and reproduction in any medium, provided the original work is properly cited.

\begin{abstract}
An optimal resource allocation strategy for MIMO relay system is considered in simultaneous wireless information and energy transfer network, where two users with multiple antennas communicate with each other assisted by an energy harvesting MIMO relay that gathers energy from the received signal by applying time switching scheme and forwards the received signal by using the harvesting energy. It is focused on the precoder design and resource allocation strategies for the system to allocate the resources among the nodes in decode-and-forward (DF) mode. Specifically, optimal precoder design and energy transfer strategy in MIMO relay channel are firstly proposed. Then, we formulate the resource allocation optimization problem. The closed-form solutions for the time and power allocation are derived. It is revealed that the solution can flexibly allocate the resource for the MIMO relay channel to maximize the sum rate of the system. Simulation results demonstrated that the performance of the proposed algorithm outperforms the traditional fixed method.
\end{abstract}

\section{Introduction}

Wireless power transfer technology, where the receiver can scavenge energy from the received signals, has recently attracted much attention in academia and industry $[1,2]$. It is a promising technology to overcome the bottleneck of energy constrained wireless networks. The nodes collect the energy from radio frequency $(\mathrm{RF})$ signals to charge their batteries by the electromagnetic radiation theory [3]. RF energy transfer can be fully controlled and fit for the scenarios with strict quality of service constraints. Meanwhile, the conventional renewable energy resources (wind/solar/tide energy) are not satisfied with these scenarios due to their intermittent and unpredictable properties [4].

The concept of simultaneous wireless information and power transfer (SWIPT) is first proposed by Varshney in [5]. In [6], Zhou et al. proposed the SWIPT architecture for receiver design and suggested two methods to distinguish information and energy: time switching (TS) and power splitting (PS). Considering cochannel interference, an optimal design is proposed for outage-energy tradeoff and rate-energy tradeoff in SWIPT [7]. In [8-10], researchers extend this concept to MIMO, OFDM, and cooperation system. Zhang Rui considers a MIMO broadcasting method for SWIPT. The optimal precoder design is proposed and the rate-energy region is obtained in MIMO scenarios [11]. Considering the limited feedback constraint, an optimal beamforming design to trade off the energy harvesting and information transfer in multiple antennas system is investigated [12]. The work in [13] proposes an amplify-andforward relaying protocol for SWIPT with TS and PS mode and investigates the influence of different parameters on the system performance. In [14], the throughput for a Gaussian relay network with energy harvesting constraint is analyzed. Ding et al. proposed several power allocation strategies to optimize the outage probability in DF cooperative network where multiple source-destination pairs communicate via a shared energy harvesting relay [15]. Combing MIMO and cooperative technology, Krikidis et al. investigates a lowcomplexity antenna switching between decoding/rectifying in order to achieve optimal outage probability with simultaneous information and energy transfer in [16].

In this paper, we focus on a general MIMO cooperative network, where two users communicated with each other 


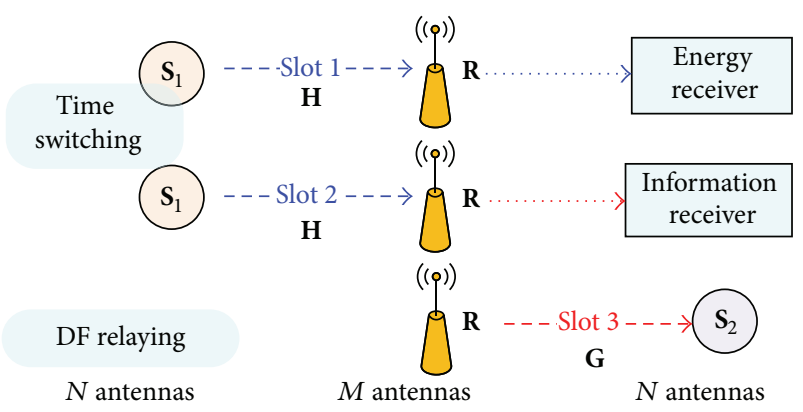

FIGURE 1: System model of MIMO relay network with energy harvesting.

via an energy harvesting relay. Specifically, each node is equipped with multiple antennas. The relaying transmission is powered by the scavenged energy from the signals sent by the users. Assuming that the battery of the relay is sufficiently large, the relay can accumulate a significant amount of power for relaying transmission. The aim of this paper is to analyze the precoder design for the source node and the relay node to optimize the system performance and study how to efficiently distribute the time resource between information transmission and power transfer. Moreover, the optimal power allocation strategies for the system are also investigated.

This paper is organized as follows. Section 2 introduces the system model and the basic notation. In Section 3, we present the proposed precoder design for DF model, Moreover, the optimal transmission ratio between energy transfer and information transmission is also derived. Simulation results and comparisons are given in Section 4, and, finally, conclusions are drawn in Section 5.

\section{System Model}

We consider a MIMO energy harvesting (EH) cooperative network with two users and a relay node as shown in Figure 1. All nodes operate in half-duplex mode. Two users are equipped with $N$ antennas and fix power supply. They cannot communicate with each other directly. They exchange the messages assisted by the energy harvesting relay node. The relay node is battery-free and equipped with $M$ antennas. It can scavenge the energy from its observation. We adopt time switching method to charge the battery. It means that the relay node harvests the energy from RF signals transmitted by the users and uses this energy to relay users' information.

The relaying protocol with energy harvesting can be stated as follows.

In the first phase (Slot 1), the source terminal transmits the signal to the energy harvesting relay node with precoder matrix $\mathbf{S}_{\mathrm{EH}} \in \mathbb{C}^{N \times N}$ for energy transfer. It is worth noting that the $\mathrm{EH}$ receiver at the relay node does not need to convert the received signal from the RF band to the baseband for scavenging the carried energy. Nevertheless, according to the law of energy conservation, it can be supposed that the total harvested RF-band power is proportional to that of the received baseband signal. The harvested energy can be normalized by the baseband symbol period from all receiving antennas at the $\mathrm{EH}$ receiver. Therefore, the scavenged power at the relay node can be presented as

$$
Q_{R}=\alpha \zeta\left\|\mathbf{H S}_{\mathrm{EH}} \mathbf{x}\right\|^{2},
$$

where $\mathbf{x} \in \mathbb{C}^{N \times 1}$ denotes the data symbol to be transmitted by source user. In addition, we assume that there is a power constraint at the transmitter across all transmitting antennas denoted as $\operatorname{tr}\left(\mathbf{S}_{R 1}\right) \leq P$, where $\mathbf{S}_{R 1}=\mathbf{S}_{\mathrm{EH}} \mathbf{x}\left(\mathbf{S}_{\mathrm{EH}} \mathbf{x}\right)^{H}$ indicates the covariance matrix of transmit signal in the first phase. $\mathbf{H} \in \mathbb{C}^{M \times N}$ denotes the channel coefficients between the relay and the source terminals; $\zeta(0<\zeta<1)$ is the energy conversion efficiency at the relay node R. $\alpha(0<\alpha<1 / 2)$ denotes the percentage of transmission time allocated to the $\mathrm{EH}$ time phase. For simplicity, it is assumed in (1) that the scavenged energy due to the background noise at the $\mathrm{EH}$ receiver is negligible and thus can be ignored.

In the second phase (Slot 2), the source terminal transmits the signal to the energy harvesting relay node with precoder matrix $\mathbf{S}_{\mathrm{ID}} \in \mathbb{C}^{N \times N}$ for information decoding (ID). The relay's detection is based on the following observation:

$$
\mathbf{y}_{R}=\left(\frac{1}{2}-\alpha\right) \mathbf{H S}_{\mathrm{ID}} \mathbf{x}+\mathbf{n}_{R},
$$

where $\mathbf{y}_{R}$ denotes the received signal at the relay node. $\mathbf{n}_{R} \in$ $\mathbb{C}^{M \times 1}$ is the additional Gaussian noise vector for the baseband signal and $\mathbf{n}_{R} \sim \mathscr{C} \mathscr{N}(0, \mathbf{I})$. The transmit power constraint should be also maintained $\left\|\mathbf{S}_{\mathrm{ID}} \mathbf{x}\right\|^{2} \leq P$. The throughput of the system within the second phase can be expressed as

$$
r_{R}=\left(\frac{1}{2}-\alpha\right) \log _{2}\left|\mathbf{I}+\mathbf{H S}_{R} \mathbf{H}^{H}\right|,
$$

where $\mathbf{S}_{R}=\mathbf{S}_{\mathrm{ID}} \mathbf{x}\left(\mathbf{S}_{\mathrm{ID}} \mathbf{x}\right)^{H}$ denotes the covariance matrix of transmit signal. We consider a DF relay protocol for the MIMO relaying network with energy harvesting. The relay node will decode the signal and reconstruct the message $\mathbf{x}_{R} \in$ $\mathbb{C}^{M \times 1}$ for the transmission in the third phase. Furthermore, the relay node simultaneously processes and forwards the received signals using the harvested energy.

It is noted that the total duration for the first and second phase is half of the total transmission of the relay system. It can maintain the consistence with the conventional relay transmission protocol that the durations of the first hop and the second hop are the same. Then, the consumed energy of the transmitter in proposed protocol is the same with conventional relay transmission protocol. Therefore, the simulation comparison in Section 4 is fair for all the mentioned algorithms.

In the third phase (Slot 3 ), the relay node broadcasts the signal with precoder matrix $S_{\mathrm{BC}} \in \mathbb{C}^{M \times M}$ by using a multiplexing strategy. The received signal $\mathbf{y}_{D}$ at the destination terminal can be presented as

$$
\mathbf{y}_{D}=\frac{1}{2} \mathbf{G S}_{\mathrm{BC}} \mathbf{x}_{R}+\mathbf{n}_{D}
$$


where $\mathbf{G} \in \mathbb{C}^{M \times N}$ denotes the channel coefficients between the relay and the destination terminal. The additional Gaussian noise at the destination terminal is $\mathbf{n}_{D} \in \mathbb{C}^{N \times 1} \sim$ $\mathscr{C} \mathcal{N}(0, \mathbf{I})$, noted that the relay node is battery-free. It needs to scavenge energy from the received signal in the first phase. Therefore, the energy constraint $(1 / 2)\left\|\mathbf{S}_{\mathrm{BC}} \mathbf{x}_{R}\right\|^{2} \leq Q_{R}$ in the third phase would be maintained. Then the data rate of the destination terminal can be expressed as

$$
r_{D}=\frac{1}{2} \log _{2}\left|\mathbf{I}+\mathbf{G S}_{D} \mathbf{G}^{H}\right|,
$$

where $\mathbf{S}_{D}=\mathbf{S}_{\mathrm{BC}} \mathbf{x}_{R}\left(\mathbf{S}_{\mathrm{BC}} \mathbf{x}_{R}\right)^{H}$ denotes the covariance matrix of broadcast signal.

Then, the rate in DF cooperative network with energy harvesting can be presented as

$$
r=\min \left(r_{R}, r_{D}\right) .
$$

In the following, we will investigate the optimal transmit precoder matrix $\mathbf{S}_{\mathrm{EH}}, \mathbf{S}_{\mathrm{ID}}$, and $\mathbf{S}_{\mathrm{BC}}$ to maximize the transported energy efficiency and information rate for the relay node. Moreover, the optimal time switching ratio $\alpha$ is also analyzed to maximize the system throughput.

\section{Problem Formulation and Optimal Solution}

In this section, we formulate the optimal problem to maximize the system throughput. Consider the three-phase MIMO link from the transmitter to the relay node and the relay node to the receiver; the optimization problem can be formulated as follows:

$$
\begin{array}{ll}
\underset{\alpha, \mathbf{S}_{\mathrm{EH}}, \mathbf{S}_{\mathrm{ID}}, \mathbf{S}_{\mathrm{BC}}}{\operatorname{maximize}} & r=\min \left(r_{R}, r_{D}\right) \\
\text { s.t. } & \frac{1}{2} \geq \alpha \geq 0 \\
& \operatorname{tr}\left(\mathbf{S}_{R 1}\right)<P \\
& \operatorname{tr}\left(\mathbf{S}_{R 2}\right)<P \\
& \frac{1}{2} \operatorname{tr}\left(\mathbf{S}_{D}\right) \leq Q_{R} .
\end{array}
$$

Since there are too many variables in the optimization problem, the energy constraint and rate in relay node with energy harvesting are nonconcave function for $\alpha$; it is difficult to solve this problem directly.

In the following, we propose dividing the original problem into four subproblems. Each subproblem can be solved efficiently by using convex optimization technology.

Consider the MIMO link of the first phase from the transmitter to the relay node; the design objective in this case is to maximize the scavenged power. Subproblem (1) can be formulated as

$$
\begin{array}{ll}
\underset{\mathbf{S}_{\mathrm{EH}}}{\operatorname{maximize}} & Q_{R}=\alpha \zeta \operatorname{tr}\left(\mathbf{H S}_{R 1} \mathbf{H}^{H}\right) \\
\text { s.t. } & \operatorname{tr}\left(\mathbf{S}_{R 1}\right) \leq P \quad \mathbf{S}_{R 1} \geq \mathbf{0} .
\end{array}
$$

Since the objective function is monotonically increasing of $\alpha$, we will focus the issue on the $\mathbf{S}_{\mathrm{EH}}$ optimal design. Applying the singular value decomposition (SVD) method, the MIMO link between transmitter and the relay node can be decoupled into several independent subchannels:

$$
\mathbf{H}=\mathbf{U} \Sigma \mathbf{V}^{H} \text {, }
$$

where $\mathbf{U} \in \mathbb{C}^{M \times L}$ and $\mathbf{V} \in \mathbb{C}^{N \times L}$ are complex unitary matrix, each of which consists of orthogonal columns with unit norm. Moreover $\boldsymbol{\Sigma}=\operatorname{diag}\left\{\sqrt{h_{1}}, \sqrt{h_{2}}, \ldots, \sqrt{h_{L}}\right\}, L=$ $\min \{M, N\}$ with the singular values in order of decreasing size. Then applying eigenvalue decomposition method $\mathbf{S}_{R 1}$ can be expressed as

$$
\mathbf{S}_{R 1}=\mathbf{V}_{R 1} \Lambda_{\mathrm{EH}} \mathbf{V}_{R 1}^{H} \text {, }
$$

where $\mathbf{V}_{R 1}=\left[\mathbf{v}_{R 1,1}, \mathbf{v}_{R 1,2}, \ldots, \mathbf{v}_{R 1, N}\right] \in \mathbb{C}^{N \times N}, \mathbf{V}_{R 1} \mathbf{V}_{R 1}^{H}=\mathbf{I}$ is unitary matrix, and $\boldsymbol{\Lambda}_{\mathrm{EH}}=\operatorname{diag}\left\{P_{1,1}, P_{1,2}, \ldots, P_{1, N}\right\}$. Then the power constraints in subproblem (1) can be rewritten as

$$
\begin{gathered}
\operatorname{tr}\left(\mathbf{S}_{R 1}\right) \leq P, \\
\sum_{n=1}^{N} P_{1, n} \leq P .
\end{gathered}
$$

Substitute (10)-(11) into subproblem (1); the objective function can be repressed as

$$
\begin{aligned}
Q_{R} & =\alpha \zeta \operatorname{tr}\left(\mathbf{H S}_{R 1} \mathbf{H}^{H}\right) \\
Q_{R} & =\alpha \zeta \sum_{n=1}^{N} P_{1, n}\left\|\widetilde{\mathbf{h}}_{n}\right\|^{2},
\end{aligned}
$$

where $\widetilde{\mathbf{h}}_{n}$ is the column element of $\mathbf{H V}_{R 1}$. Apparently, in order to maximize the scavenged power $Q_{R}$, we just need to find the maximum value of all $\left\|\widetilde{\mathbf{h}}_{n}\right\|^{2}$ and allocate all power to this subchannel. The inequality can be expressed as

$$
Q_{R}=\alpha \zeta \sum_{n=1}^{N} P_{1, n}\left\|\widetilde{\mathbf{h}}_{n}\right\|^{2} \leq \alpha \zeta P\left\|\widetilde{\mathbf{h}}_{1}\right\|^{2}
$$

Considering (9), the value of $\mathbf{v}_{R 1,1}$ in $\mathbf{V}_{R 1}$ is the first column of $\mathbf{V}$ corresponding to the largest singular value of $\mathbf{H}$, which is $\sqrt{h_{1}}$. In this scenario, the equality of (13) would hold. Therefore, the optimal solution for subproblem (1) can be obtained as

$$
\begin{gathered}
\mathbf{S}_{R 1}=P \mathbf{v}_{R 1,1} \mathbf{v}_{R 1,1}^{H} \\
\mathbf{S}_{\mathrm{EH}}=\sqrt{P} \mathbf{v}_{R 1,1} .
\end{gathered}
$$

And the optimal harvested power at the relay node is

$$
Q_{R}=\alpha \zeta P h_{1} .
$$

Consider the MIMO link of the second phase from the transmitter to the relay node; the design objective in this 
case is to maximize the data rate over the MIMO channel. Subproblem (2) can be formulated as

$$
\begin{array}{ll}
\underset{\mathbf{S}_{\mathrm{ID}}}{\operatorname{maximize}} & r_{R}=\left(\frac{1}{2}-\alpha\right) \log _{2}\left|\mathbf{I}+\mathbf{H} \mathbf{S}_{R 2} \mathbf{H}^{H}\right| \\
\text { s.t. } & \operatorname{tr}\left(\mathbf{S}_{R 2}\right) \leq P \quad \mathbf{S}_{R 2} \geq \mathbf{0} .
\end{array}
$$

It is a typical optimization problem of MIMO system [17]. Applying the SVD method, the optimal solution can be obtained as follows:

$$
\begin{aligned}
& \mathbf{S}_{R 2}=\mathbf{V} \Lambda_{\mathrm{ID}} \mathbf{V}^{H} \\
& \mathbf{S}_{\mathrm{ID}}=\mathbf{V} \Lambda_{\mathrm{ID} \text { ID }}^{1 / 2}
\end{aligned}
$$

where $\Lambda_{\mathrm{ID}}=\operatorname{diag}\left\{P_{2,1}, P_{2,2}, \ldots, P_{2, L}\right\}, L=\min \{M, N\}$. Apparently, the water-filling power allocation solution would maximize the data rate of the MIMO channel. It can be stated as follows:

$$
P_{2, l}=\left[\lambda-\frac{1}{h_{l}}\right]^{+} \quad l=1,2, \ldots, L,
$$

where $\lambda$ is the constant water level that makes the sum power of all subchannels satisfy the power constraint of $\sum_{l=1}^{L} P_{2, l} \leq$ $P$. Therefore, the sum rate of the system within the second phase can be presented as

$$
r_{R}=\left(\frac{1}{2}-\alpha\right) \sum_{l=1}^{L} \log _{2}\left(1+P_{2, l} h_{l}\right) .
$$

Consider the MIMO link of the third phase from the relay node to the receiver; the design objective in this case is also to maximize the data rate over the MIMO channel. The subproblem can be formulated as

$$
\begin{array}{ll}
\underset{\mathbf{S}_{\mathrm{ID}}}{\operatorname{maximize}} & r_{D}=\frac{1}{2} \log _{2}\left|\mathbf{I}+\mathbf{G S}_{D} \mathbf{G}^{H}\right| \\
\text { s.t. } & \frac{1}{2} \operatorname{tr}\left(\mathbf{S}_{D}\right) \leq Q_{R}, \quad \mathbf{S}_{D} \succeq \mathbf{0} .
\end{array}
$$

Supposing that $Q_{R}$ is fixed, the optimal solution is the same as in the second phase, which has the following form:

$$
\begin{gathered}
\mathbf{S}_{D}=\mathbf{V}_{G} \boldsymbol{\Lambda}_{\mathrm{BC}} \mathbf{V}_{G}^{H}, \\
\mathbf{S}_{\mathrm{BC}}=\mathbf{V}_{G} \boldsymbol{\Lambda}_{\mathrm{BC}}^{1 / 2},
\end{gathered}
$$

where $\mathbf{V}_{G} \in \mathbb{C}^{M \times L}$ is the unitary matrix obtained from the SVD of $\mathbf{G}$, presented as $\mathbf{G}=\mathbf{U}_{G} \boldsymbol{\Sigma}_{\mathrm{BC}} \mathbf{V}_{G}^{H}$, where $\boldsymbol{\Sigma}_{\mathrm{BC}}=$ $\operatorname{diag}\left\{\sqrt{g_{1}}, \sqrt{g_{2}}, \ldots, \sqrt{g_{L}}\right\}$ with the singular values in order of decreasing size. $\boldsymbol{\Lambda}_{\mathrm{BC}}=\operatorname{diag}\left\{p_{R, 1}, p_{R, 2}, \ldots, p_{R, L}\right\}$ denotes the power values assigned to the subchannels. Then, just like the case in the second phase, the optimal power allocation for the subchannels in the MIMO channel of the third phase can be given as

$$
P_{R, l}=\left[\mu-\frac{1}{g_{l}}\right]^{+}, \quad l=1,2, \ldots, L
$$

where $\mu$ is the constant water level that makes the sum power of all subchannels satisfy the power constraint of $(1 / 2) \sum_{l=1}^{L} P_{R, l} \leq Q_{R}$. And the sum rate can be presented as

$$
r_{R}=\frac{1}{2} \sum_{l=1}^{L} \log _{2}\left(1+P_{R, l} g_{l}\right) .
$$

Until now, the original problem can be reformulated as

$$
\begin{array}{ll}
\underset{\alpha}{\operatorname{maximize}} & r=\min \left(r_{R}, r_{D}\right) \\
\text { s.t. } & \frac{1}{2} \geq \alpha \geq 0 \\
& \frac{1}{2} \sum_{l=1}^{L} P_{R, l} \leq \alpha \zeta P h_{1} .
\end{array}
$$

Due to the rate constraint of the DF protocol, the optimal point of the problem would be obtained when the rates of two hops are equal. In other words, the following formula must be established:

$$
\begin{gathered}
\frac{1}{2} \sum_{l=1}^{L} \log _{2}\left(1+P_{R, l} g_{l}\right) \\
=\left(\frac{1}{2}-\alpha\right) \sum_{l=1}^{L} \log _{2}\left(1+P_{2, l} h_{l}\right) \\
\frac{1}{2} \sum_{l=1}^{L} P_{R, l}=\alpha \zeta P h_{1} .
\end{gathered}
$$

Since $h_{l}, g_{l}$ is known within each transmission period, the optimal $P_{2, l}^{*}$ can be calculated through (18). Then we can set $\sum_{l=1}^{L} \log _{2}\left(1+P_{2, l}^{*} h_{l}\right)$ as a fixed value $\widetilde{r}_{R}$. Substitute (22), (26) into (25); we can get a function with only one variable $\mu$ as follows:

$$
\begin{aligned}
& \frac{1}{2} \sum_{l=1}^{L} \log _{2}\left(1+\left(\mu-\frac{1}{g_{l}}\right)^{+} g_{l}\right) \\
& \quad=\left(\frac{1}{2}-\frac{(1 / 2) \sum_{l=1}^{L}\left(\mu-1 / g_{l}\right)^{+}}{\zeta P h_{1}}\right) \widetilde{r}_{R} .
\end{aligned}
$$

It is a nonlinear logarithmic equation. It can be solved efficiently by using library function "fzero" in Matlab. While the optimal $\mu^{*}$ is found, the optimal $\alpha^{*}$ can be given as

$$
\alpha^{*}=\frac{(1 / 2) \sum_{l=1}^{L}\left(\mu^{*}-1 / g_{l}\right)^{+}}{\zeta P h_{1}} \text {. }
$$

In summary, we proposed an optimal transmission strategy for MIMO relay network with energy harvesting. The whole transmission is divided into three phases. For the first phase, an energy beamforming precoder matrix to maximize the scavenged power at the relay node is proposed. For the second and third phase, we proposed a spatial multiplexing precoder matrix to obtain the maximum data rate. Moreover, the optimal time switching ratio is also investigated to balance the power transfer and information transmission. 


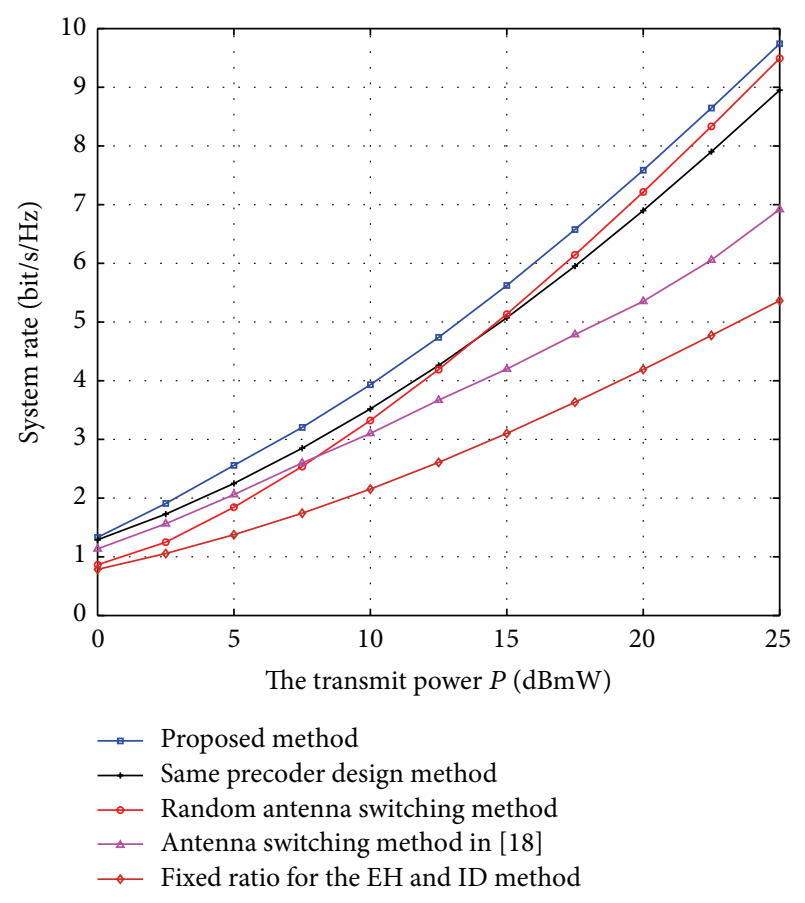

FIGURE 2: Achievable sum rate of the SWIPT relay network for different transmission strategy.

\section{Simulation Results}

In this section, the performance of MIMO relay network with energy harvesting would be investigated. For comparison, we adopt four reference methods: (1) the same precoder matrix for the first and second phase with optimal time switching ratio; (2) the optimal precoder matrix for the first and second phase with fixed time switching ratio; (3) random antenna switching for energy harvesting and information transmission; and (4) antenna switching scheme in [18]. Two users and relay node are distributed in a one-dimensional region. Suppose that two terminals are separated in a normalized distance. Denote the distance between transmitters and relay node as $d$ and for destination terminal as $(1-d)$. Suppose the large scale path loss factor $\phi=3$; the channel coefficients between relay and terminals would have additional large scale path loss $\Omega_{1}=d^{-3}, \Omega_{2}=(1-d)^{-3}$, respectively. We set the noise power as $-60 \mathrm{dBmW}$.

First, we evaluate the system throughput of all the mentioned methods. Suppose that each node equipped 4 antennas. The relay node is in the middle of the source terminal and destination terminal. Figure 2 shows the performance of all the mentioned methods under different transmit power. It shows that the proposed solution outperforms other methods. For method (1), the power loss due to the energy beamforming precoder matrix design is not optimal. The power transfer through the subchannels with low coefficients would waste the source energy; the performance gap especially would be apparently in high transmit power region. For method (2), the fixed ratio for energy transfer and information would not balance the rate and available energy

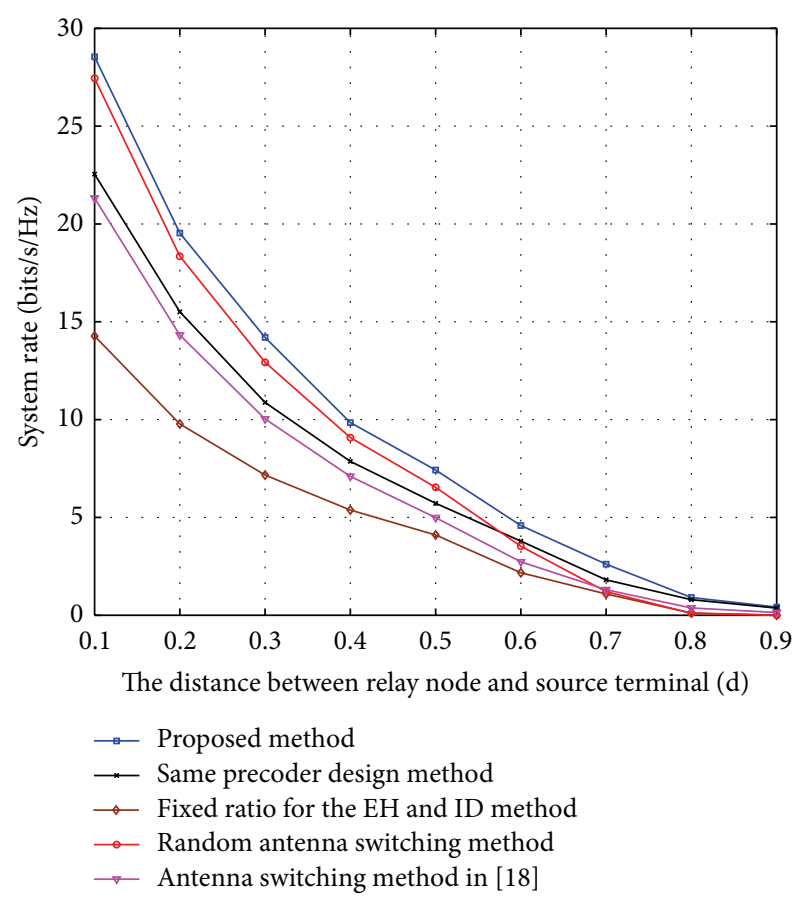

FIGURE 3: Achievable sum rate of the SWIPT relay network for different relay position (total transmit power $P=20 \mathrm{dBmW}$ ).

for the third phase. For example, if the power scavenged in the first phase with fixed splitting ratio is small. Then, it cannot surpport the information transmission of the third phase, since the target rate of third phase is constraint by the second transmission phase with the fixed splitting ratio. For method (3), it was proposed to divide the antennas into energy harvesting mode and information received mode with optimal precoder design during the first phase. In low transmit power region, the scavenged power is the bottleneck for the system throughput. In high transmit power region, since each antenna would have the good channel condition, the performance is close to the proposed method. For method (4), it was proposed to select the proper antenna working at energy harvesting mode or information decoding mode by analyzing the channel condition of each antenna. But the algorithm did not consider the precoder design to improve the system performance. The performance loss would be increased especially in high transmit power region.

Next, considering the total transmit power is $20 \mathrm{dBmW}$, we investigate the influence on the rate with the different distance between relay node and users. Figure 3 shows that the performance would decrease when the distance between source terminals and relay node increases. It is because that the harvested energy and the received signal strength at the relay node would be decreased due to the large path loss. Moreover, the proposed method outperforms other solutions. Random antenna switching method would decrease the performance at the long distance due to the large path loss. It demonstrated that the proposed method can balance the harvested energy and the rates of the two hops to maximum system throughput in different scenarios. 


\section{Conclusions}

In this paper, an optimal transmission strategy for DF MIMO relay network with energy harvesting is investigated. It is proposed to design optimal precoders specifically for energy harvesting and information transmission. For energy harvesting, an energy beamforming matrix is designed to scavenge maximum power during the finite time. For information transmission, traditional SVD method is adopted. Moreover, the optimal ratio of the time between energy harvesting and information transmission is also derived. Numerical results show that the proposed method outperforms other traditional methods. In future work, optimal strategy with QoS constraint for multiple relay nodes would be further investigated.

\section{Conflict of Interests}

The authors declare that there is no conflict of interests regarding the publication of this paper.

\section{Acknowledgments}

This work was partly supported by the National Natural Science Foundation of China $(61340025,61461029)$, the Natural Science Foundation of Jiangxi Province (20114ACE00200, 20142BAB217005, and 20142BBE50046), Technology Foundation of Department of Education in Jiangxi Province (no. GJJ13062), and China/Jiangxi Postdoctoral Science Foundation Funded Project (nos. 2013MT541875, 2014MT561879, 2013KY007, and 2014KY046).

\section{References}

[1] A. Kurs, A. Karalis, R. Moffatt, J. D. Joannopoulos, P. Fisher, and M. Soljačić, "Wireless power transfer via strongly coupled magnetic resonances," American Association for the Advancement of Science: Science, vol. 317, no. 5834, pp. 83-86, 2007.

[2] U. Olgun, C.-C. Chen, and J. L. Volakis, "Wireless power harvesting with planar rectennas for $2.45 \mathrm{GHz}$ RFIDs," in Proceedings of the URSI International Symposium on Electromagnetic Theory (EMTS '10), pp. 329-331, Berlin, Germany, October 2010.

[3] M. Mi, M. H. Mickle, C. Capelli, and H. Swift, "RF energy harvesting with multiple antennas in the same space," IEEE Antennas and Propagation Magazine, vol. 47, no. 5, pp. 100-106, 2005.

[4] J. Xu and R. Zhang, "Throughput optimal policies for energy harvesting wireless transmitters with non-ideal circuit power," IEEE Journal on Selected Areas in Communications, vol. 32, no. 2, pp. 322-332, 2014.

[5] L. R. Varshney, "Transporting information and energy simultaneously," in Proceedings of the IEEE International Symposium on Information Theory (ISIT '08), pp. 1612-1616, Toronto, Canada, July 2008.

[6] X. Zhou, R. Zhang, and C. K. Ho, "Wireless information and power transfer: architecture design and rate-energy tradeoff," IEEE Transactions on Communications, vol. 61, no. 11, pp. 47544767, 2013.
[7] L. Liu, R. Zhang, and K.-C. Chua, "Wireless information transfer with opportunistic energy harvesting," IEEE Transactions on Wireless Communications, vol. 12, no. 1, pp. 288-300, 2013.

[8] R. Feng, Q. Li, Q. Zhang, and J. Qin, "Robust secure transmission in MISO simultaneous wireless information and power transfer system," IEEE Transactions on Vehicular Technology, vol. 64, no. 1, pp. 400-405, 2014.

[9] L. Liu, R. Zhang, and K. C. Chua, "Wireless information and power transfer: a dynamic power splitting approach," IEEE Transactions on Communications, vol. 61, no. 9, pp. 3990-4001, 2013.

[10] D. W. K. Ng, E. S. Lo, and R. Schober, "Energy-efficient resourceallocation in multiuser ofdm systems with wireless information andpower transfer," IEEE Transactions on Wireless Communications, vol. 12, no. 12, pp. 6352-6370, 2013.

[11] R. Zhang and C. K. Ho, "MIMO broadcasting for simultaneous wireless information and power transfer," IEEE Transactions on Wireless Communications, vol. 12, no. 5, pp. 1989-2001, 2013.

[12] X. Chen, C. Yuen, and Z. Zhang, "Wireless energy and information transfer tradeoff for limited-feedback multiantenna systems with energy beamforming," IEEE Transactions on Vehicular Technology, vol. 63, no. 1, pp. 407-412, 2014.

[13] A. A. Nasir, X. Zhou, S. Durrani, and R. A. Kennedy, "Relaying protocols for wireless energy harvesting and information processing," IEEE Transactions on Wireless Communications, vol. 12, no. 7, pp. 3622-3636, 2013.

[14] C. Huang, R. Zhang, and S. Cui, "Throughput maximization for the gaussian relay channel with energy harvesting constraints," IEEE Journal on Selected Areas in Communications, vol. 31, no. 8, pp. 1469-1479, 2013.

[15] Z. Ding, S. M. Perlaza, I. Esnaola, and H. V. Poor, "Power allocation strategies in energy harvesting wireless cooperative networks," IEEE Transactions on Wireless Communications, vol. 13, no. 2, pp. 846-860, 2014.

[16] I. Krikidis, S. Sasaki, S. Timotheou, and Z. Ding, "A low complexity antenna switching for joint wireless information and energy transfer in MIMO relay channels," IEEE Transactions on Communications, vol. 62, no. 5, pp. 1577-1587, 2014.

[17] S. Vishwanath, N. Jindal, and A. Goldsmith, "Duality, achievable rates, and sum-rate capacity of Gaussian MIMO broadcast channels," IEEE Transactions on Information Theory, vol. 49, no. 10, pp. 2658-2668, 2003.

[18] I. Krikidis, S. Sasaki, and S. Timotheou, "Simultaneous wireless information and energy transfer for MIMO relay channel with antenna switching," in Proceedings of the IEEE International Conference on Communications (ICC '14), pp. 3800-3805, Sydney, Australia, June 2014. 

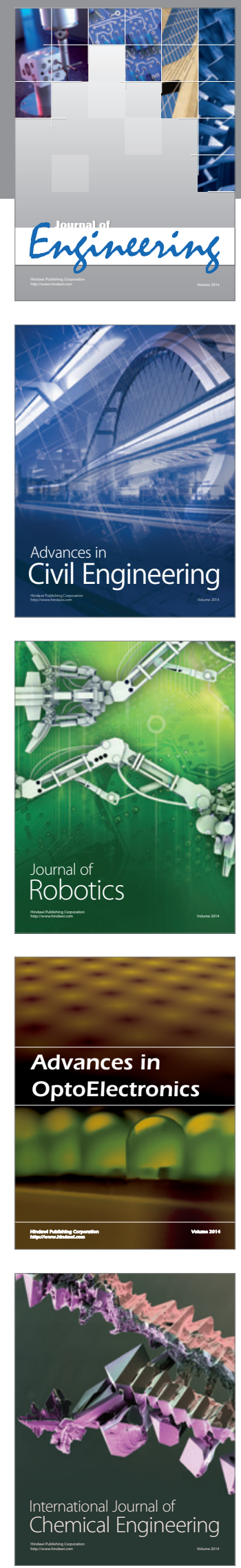

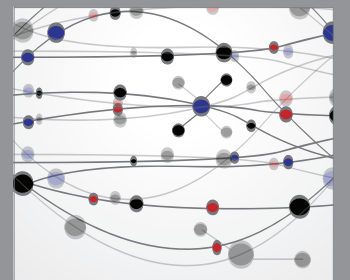

The Scientific World Journal
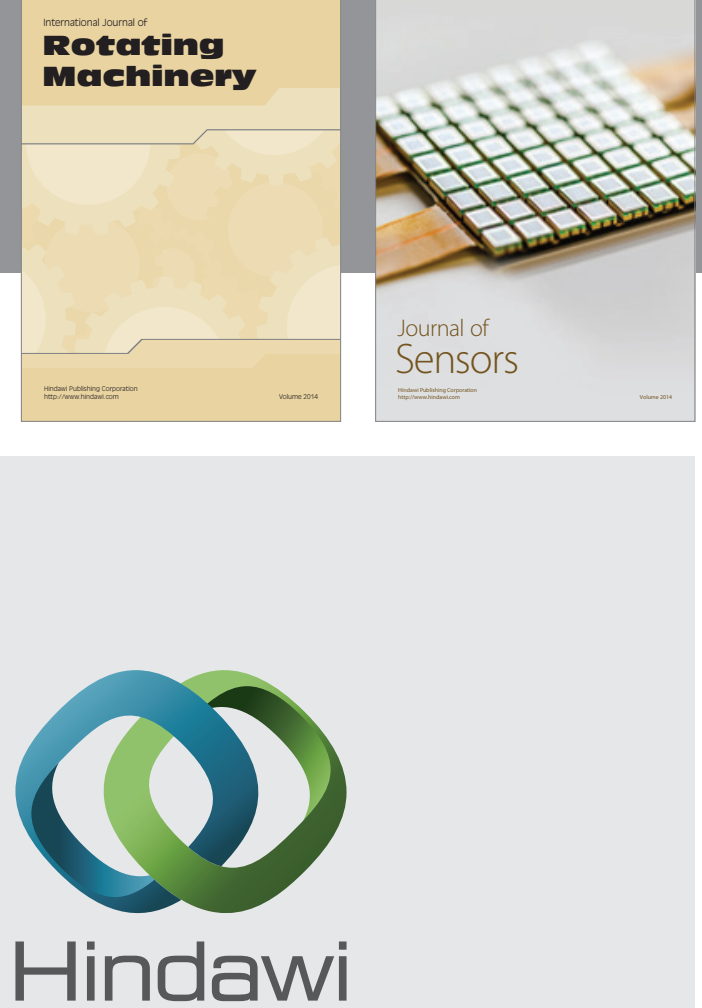

Submit your manuscripts at http://www.hindawi.com
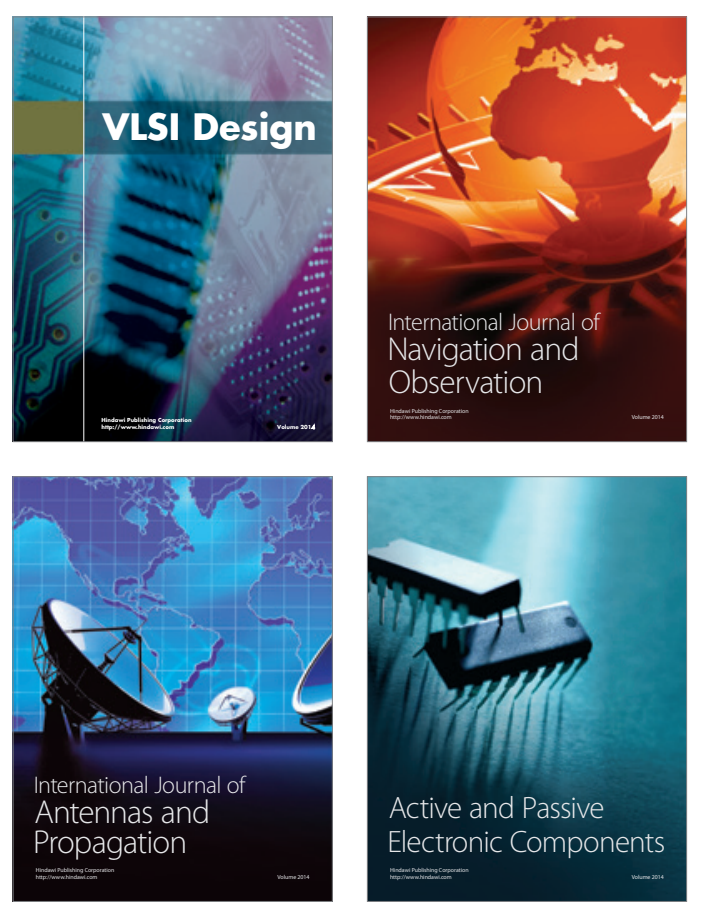
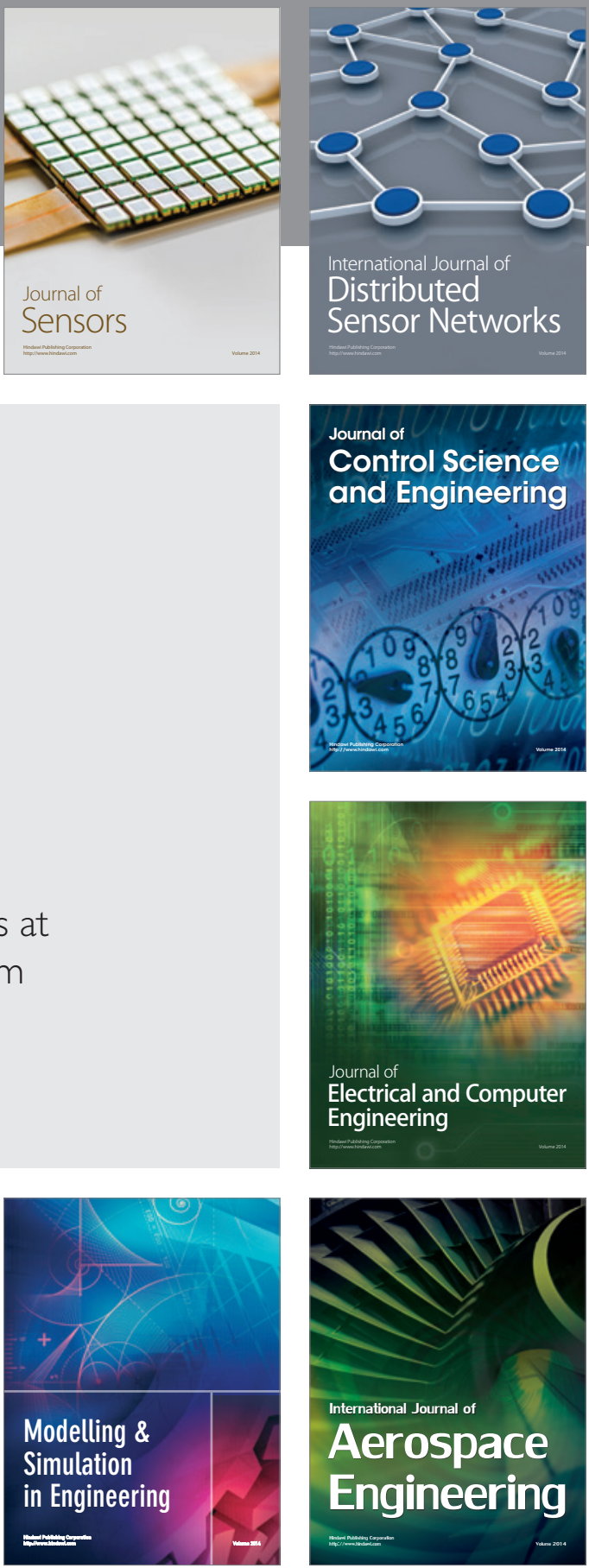

Journal of

Control Science

and Engineering
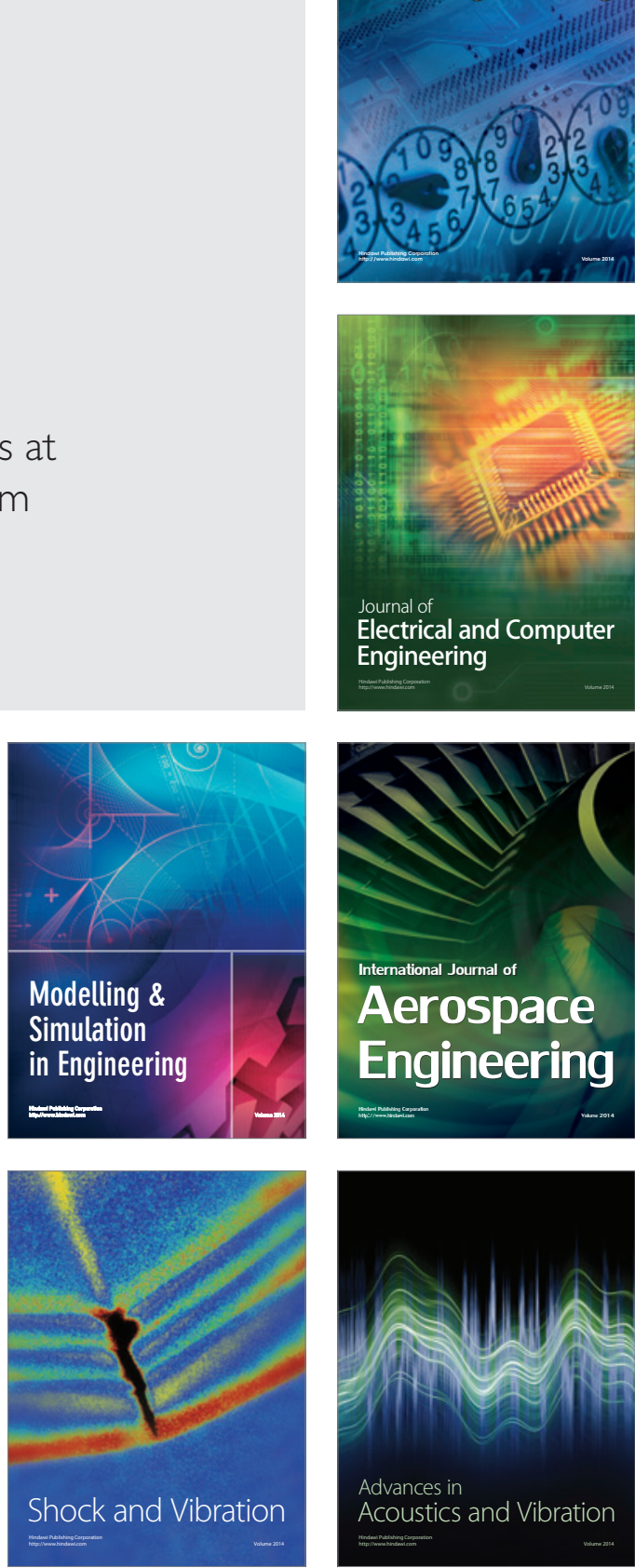